\title{
Correction to: Time to positivity of Klebsiella pneumoniae in blood culture as prognostic indicator for pediatric bloodstream infections
}

\author{
Jie Cheng ${ }^{1,2} \cdot$ Guangli Zhang ${ }^{3} \cdot$ Qingyuan $\mathrm{Li}^{1,2} \cdot$ Huiting $\mathrm{Xu}^{1,2}$. Qinghong $\mathrm{Yu}^{1,2} \cdot \mathrm{Qian}_{\mathrm{Yi}} \mathrm{i}^{1,2} \cdot$ Siying Luo ${ }^{1,2}$. \\ Yuanyuan $\mathrm{Li}^{3}$ - Xiaoyin $\mathrm{Tian}^{3}$. Dapeng Chen ${ }^{4}$ - Zhengxiu Luo ${ }^{3}$
}

Published online: 2 June 2020

(C) Springer-Verlag GmbH Germany, part of Springer Nature 2020

\section{Correction to: European Journal of Pediatrics https://doi.org/10.1007/s00431-020-03675-8}

The authors regrets that there is a typo error on the Abbreviation section of their published paper. "Area under the curve" should have been abbreviated to "AUC" instead of "A". The authors have requested that this be noted. The original article has been corrected.

The online version of the original article can be found at https://doi.org/ $10.1007 / \mathrm{s} 00431-020-03675-8$

Zhengxiu Luo

luozhengxiu816@163.com

Jie Cheng

1715777973@qq.com

Guangli Zhang

840068283@qq.com

Qingyuan Li

liqinyuan0713@163.com

Huiting Xu

xhtalizee@163.com

Qinghong Yu

568788339@qq.com

Qian Yi

524363374@qq.com

Siying Luo

313029909@qq.com
Yuanyuan Li

liyuanyuan886@qq.com

Xiaoyin Tian

1176794974@qq.com

Dapeng Chen

476538577@qq.com

Chongqing Key Laboratory of Pediatrics, Chongqing 400014, China

2 Department of Children's Hospital of Chongqing Medical University of Education; Ministry of Education Key Laboratory of Child Development and Disorders; National Clinical Research Center for Child Health and Disorders; China International Science and Technology Cooperation base of Child development and Critical Disorders, Children's Hospital of Chongqing Medical University, Chongqing 400014, China

3 Department of Respiratory Medicine, Children's Hospital of Chongqing Medical University, Chongqing 401122, China

4 Department of Clinical Laboratory Center, Children's Hospital of Chongqing Medical University, Chongqing 400014, China 